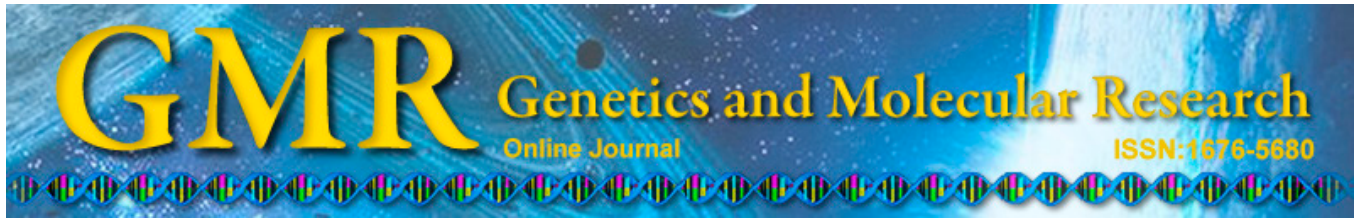

\title{
Association between 1p13.3 genomic markers and coronary artery disease: a meta-analysis involving patients and controls
}

\author{
J. Guo ${ }^{1,2}$, Y.X. Luo ${ }^{1,2}$, L.X. Tao ${ }^{1}$ and X.H. Guo ${ }^{1,2}$ \\ ${ }^{1}$ School of Public Health, Capital Medical University, Youanmen, Beijing, China \\ ${ }^{2}$ Beijing Municipal Key Laboratory of Clinical Epidemiology, Youanmen, \\ Beijing, China \\ Corresponding author: X.H. Guo \\ E-mail: guojin85827501@163.com
}

Genet. Mol. Res. 14 (3): 9092-9102 (2015)

Received January 4, 2015

Accepted May 18, 2015

Published August 7, 2015

DOI http://dx.doi.org/10.4238/2015.August.7.18

\begin{abstract}
Recently, genome-wide association studies on cardiovascular disease identified a series of associated single nucleotide polymorphisms in an intergenic region of chromosome $1 \mathrm{p} 13.3$. We investigated the association of this locus with cardiovascular disease in 13 case-control studies and undertook a meta-analysis for effect size, heterogeneity, publication bias, and strength of evidence. English and Chinese language articles were screened for the association of $1 \mathrm{p} 13.3$ single nucleotide polymorphisms with coronary heart/artery disease or myocardial infarction as primary outcomes. The included articles provided race, numbers of participants, and the data necessary to compute an odds ratio. Articles were excluded if other outcomes were reported or 1 p13.3 single nucleotide polymorphisms were not included. Thirtyfive articles were initially identified and 12 were eventually included in the meta-analysis. rs599839 and rs646776, representing the $1 \mathrm{p} 13.3$ locus, were genotyped in 13 case-control studies involving a total of 17,766 patients and 20,272 controls. For rs599839 (11 data sets), using a random-effect model, the summary odds ratio was 1.17 (95\% con-
\end{abstract}


fidence interval $=1.07-1.28, \mathrm{P}=0.0001$ ). For rs646776 (4 data sets), using a fixed-effects model, the summary odds ratio was $1.13(95 \%$ confidence interval $=1.06-1.21, \mathrm{P}=0.0001)$. This broad replication provided unprecedented evidence for an association between genetic variants at chromosome $1 \mathrm{p} 13.3$ and the risk of cardiovascular disease.

Key words: $1 \mathrm{p} 13.3$; Coronary artery disease; Genetic variants; Meta-analysis

\section{INTRODUCTION}

Cardiovascular disease (CVD) is the main cause of death and disability-adjusted life years worldwide, with increasing incidence and prevalence in low- and middle-income countries (Teo et al., 2009). By 2020, over $80 \%$ of global CVD cases will be located in these countries, with the largest burden occurring in the two largest countries, China and India, as they rapidly urbanise (Teo et al., 2009). Non-modifiable risk factors include increasing age, male gender, and heredity. Modifiable risk factors include smoking, hypertension, dyslipidemia, obesity, physical inactivity, and diabetes (Hobbs, 2004; van Wyk et al., 2005; Palomaki et al., 2010). Recently, biomarkers (e.g., C-reactive protein) have been integrated with traditional risk factors to predict CVD events, and molecular markers hold further promise (Wang et al., 2006).

In 2007, genome-wide association studies (GWASs) identified a series of single nucleotide polymorphisms (SNPs) associated with CVD in an intergenic region of chromosome 9p21, near the $C D K N 2 A$ and $C D K N 2 B$ genes (Helgadottir et al., 2007; McPherson et al., 2007). The second most replicated region for risk of coronary artery disease (CAD) is located near 1p13.3. A variant within or near the $1 \mathrm{p} 13.3$ region is associated with lower risk of both CAD and myocardial infarction (MI) (Wellcome Trust Case Control Consortium, 2007; Samani et al., 2007; Myocardial Infarction Genetics (MIGen) Consortium et al., 2009; Aulchenko et al., 2009; Muendlein et al., 2009; Ellis et al., 2011) primarily through its association with lowdensity lipoprotein (LDL) and cholesterol serum levels (Kathiresan et al., 2008a,b; Samani et al., 2008; Sandhu et al., 2008; Willer et al., 2008; Karvanen et al., 2009); lower levels of LDL, LDL triglycerides, and ApoB; and an increased radius of LDL particles (Aulchenko et al., 2009; Linsel-Nitschke et al., 2010; Kleber et al., 2010). Two leading SNPs mapping at this locus, rs646776T/C and rs599839A/G, explain 1\% of the genetic variation in circulating LDL cholesterol (LDL-C) levels and rare alleles are linked to reduced LDL-C levels (Willer et al., 2009).

In recent years, GWAS have provided a series of valuable data on the genetic susceptibility to CAD. Some regions are now used as predictive of incident CAD; however, whether these genetic risk regions influence disease progression in patients with CAD remains largely unknown. In fact, only a small proportion of the CAD population has been explained in Asia. For every traditional cohort or case-control study, the power to discover an association of statistical significance is low. To increase the power, we applied metaanalysis to pool data across all published and multiple unpublished articles for CAD and MI. In this study, we aimed to investigate the association between risk variants at $1 \mathrm{p} 13.3$ and $\mathrm{CAD}$ in different populations, and to describe in detail the structure and potential functioning of this region. 


\section{MATERIAL AND METHODS}

\section{Study populations}

We systematically searched PubMed, Blackwell, Biosis Previews, Cochrane Library, China National Knowledge Infrastructure (CNKI), Chinese Biomedical (CBM), and Wan Fang database (China) for relevant articles published in different languages up to February 2014. We used the search term " $1 \mathrm{p} 13$ and cardiovascular disease, coronary artery disease, acute myocardial infarction, myocardial infarction, structural heart disease or coronary atherosclerosis" in this study. Other eligible studies were identified by a series of references cited in published articles.

The inclusion criteria of this meta-analysis were as follows: a) inclusion of patients with a diagnosis that contained primary outcomes of coronary heart disease, MI, or CAD and without other related diseases; b) description of the association of SNPs in 1p13.3 polymorphisms with CAD risk; c) race and number of affected and unaffected participants reported; d) description of the genotyping method; and e) provision of genotype frequency and the odds ratio (OR) with confidence intervals (CIs) or data sufficient to compute it. If more than a single outcome was reported, the best-described phenotype was chosen. Several included articles reported consortium results with multiple independent populations. These populations were listed as separate data sets; all data were extracted independently by two reviewers according to the inclusion criteria. All the consistent datasets by reviewers were Samani et al., 2007; Huang et al., 2008; Muendlein et al., 2009; MIGen Consortium, 2009; Wang et al., 2011; Xie et al., 2011; Guo et al., 2011; Ellis et al., 2011; Gigante et al., 2012; Esparragón et al., 2012; Lee et al., 2013. Discrepancies were recorded and solved by discussion with a third reviewer. The information was extracted from all eligible studies: patients with a diagnosis of disease not related to CAD, and SNPs not in the 1 p13.3 region.

Overall, we studied subjects collected in 13 different studies across Europe, Asia, and Oceania. The large majority of patients had CAD as defined by the MIGen study (N $=2967$ ). The remaining patients had evidence of CAD based on either a revascularization procedure or anginal symptoms with a positive stress test. Details of each study are given in Table 1.

Not all researchers used the same 1p13.3 SNPs, and most articles reported results for multiple SNPs (uniquely identified by their rs number). As a proof-of-principle analysis, a number of SNPs in the 1p13.3 region with known association to CAD and MI were analyzed initially. Specifically, we examined two SNPs that were reported as lead SNPs in the first publications by the Wellcome Trust Case Control Consortium (2011) study (rs599839) and by Muendlein et al. (2009) (rs646776).

\section{Genotyping}

Different genotyping platforms were used across studies. An analysis restricted only to SNPs genotyped on all platforms would have been severely limited. For instance, the estimated overlap between the Affymetrix Genome Wide Human SNP Array 6.0 and the Illumina Human- $1 \mathrm{~mol} / \mathrm{L}$ chip is only about 250,000 SNPs. To allow for combined analyses across different platforms, missing SNPs were imputed for each study. 


\section{Statistical analysis}

For each data set, the observed genotype frequencies in controls were compared with expected frequencies based on Hardy-Weinberg equilibrium (HWE, $\chi^{2}$ test with two degrees of freedom). All $\mathrm{P}$ values were two-sided at the $\mathrm{P}=0.05$ level. After upload of summary data and centralized quality control, a meta-analysis across all studies was performed separately for each SNP. Depending on the heterogeneity between studies, fixed- or random-effect models were calculated, and outlying studies excluded. Summary ORs and corresponding 95\%CIs were derived (by reanalysis when possible) and summarized using random-effect modeling weighted by the total variance of each data set (STATA). Subgroup differences were compared using the Q-test for heterogeneity for each covariate separately. Publication bias was examined by performing a cumulative effect analysis. Wider ranges of these summary ORs indicated the potential for publication bias.

\section{RESULTS}

\section{Literature search and databases for meta-analysis}

A total of 31 articles were identified in the initial search with the medical subject heading terms. After screening the abstracts or full contents, 19 articles were excluded ( 8 were not related to CAD, 9 were not about SNPs in 1p13, and 2 were republished studies). Finally, 12 studies (17,766 patients and 20,272 controls) were included in this meta-analysis. Of these, 2 studies were performed in Oceania (Ellis et al., 2011), 7 in Europe and America (Samani et al., 2007; Muendlein et al., 2009; MIGen Consortium et al., 2009; Wang et al., 2011; Gigante et al., 2012; Esparragón et al., 2012), and 4 in Asia (Huang et al., 2008; Xie et al., 2011; Guo et al., 2011; Lee et al., 2013) (Figure 1 and Table 1). All genotype frequencies of studies conformed to HWE in the control groups.

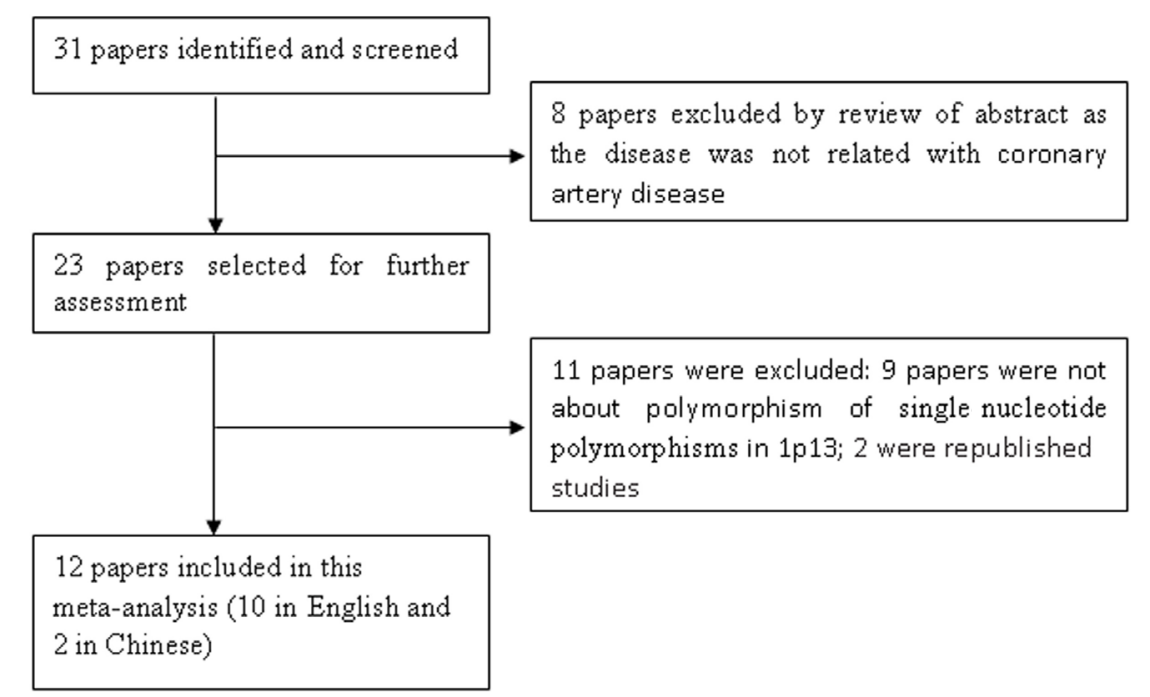

Figure 1. Paper identification and exclusion. 


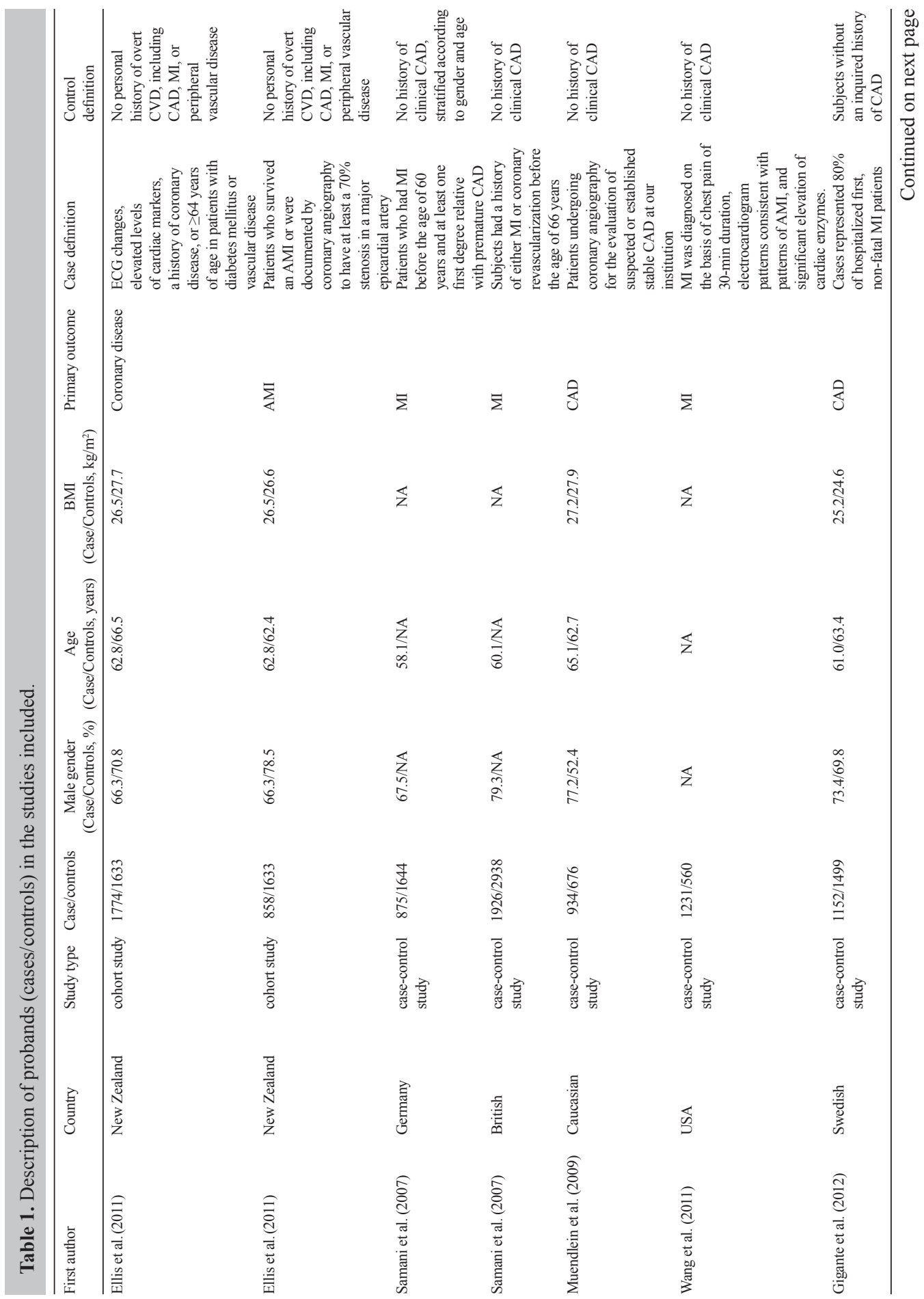




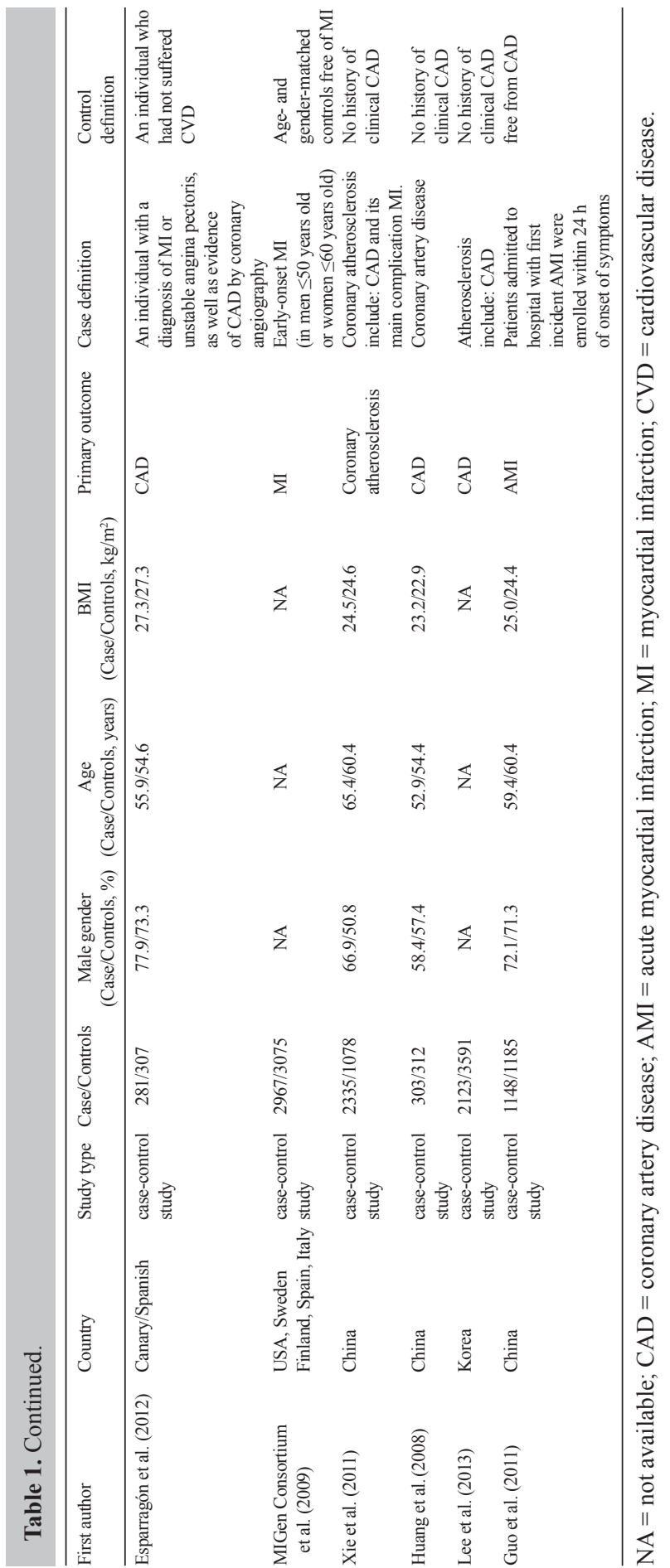




\section{Quantitative synthesis}

The results of all the populations for our proof-of-principle analysis are shown in Figures 2 and 3. For rs599839 (11 data sets), heterogeneity was checked separately using the Q statistic $(\mathrm{Q}=32.4 ; I=69.2 \% ; \mathrm{P}<0.001)$. Therefore, a random-effect model was used, and the summary OR was $1.17(95 \% \mathrm{CI}=1.07-1.28, \mathrm{P}=0.0001)$. For rs646776 (4 data sets), heterogeneity was checked separately using the $\mathrm{Q}$ statistic $\left(\mathrm{Q}=7.4 ; P^{2}=59.5 \% ; \mathrm{P}=0.060\right)$. For this analysis, a fixed-effect model was used; the summary $\mathrm{OR}$ was $1.13(95 \% \mathrm{CI}=1.06-1.21, \mathrm{P}=0.0001)$.

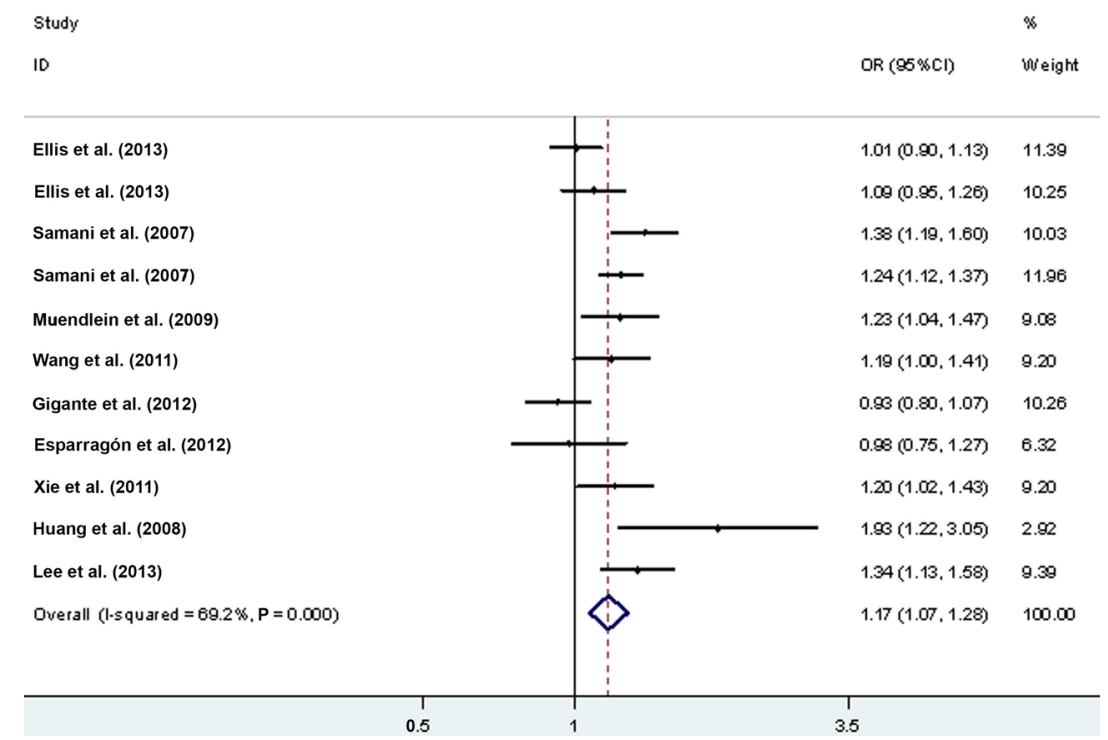

Figure 2. Forest plots for single nucleotide polymorphism rs599839 (risk allele =A); the random effect model was calculated. Heterogeneity between the studies is indicated by $I^{2}$. CI $=$ confidence interval; $\mathrm{OR}=$ odds ratio.

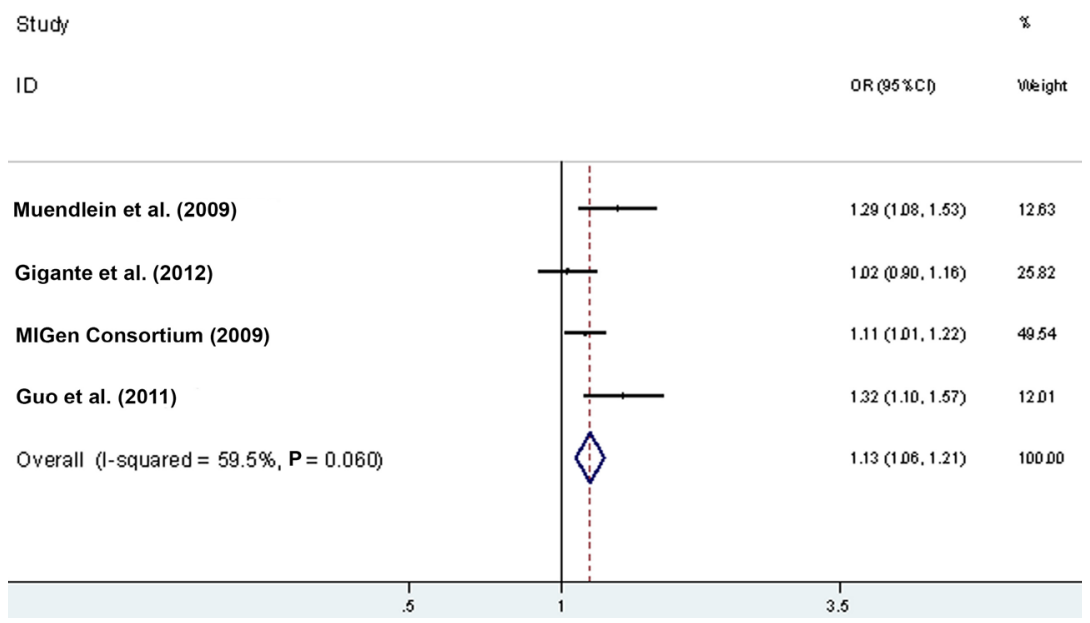

Figure 3. Forest plots for single nucleotide polymorphism rs646776 (risk allele $=\mathrm{C}$ ); the fixed-effect model was calculated. Heterogeneity between the studies is indicated by $R^{2}$. CI $=$ confidence interval; $\mathrm{OR}=$ odds ratio. 
In subgroup analyses, the A allele of SNP rs599839 was also found to be significantly associated with CAD in European and Asian populations (Table 2). The ORs were significantly different between Asians (4 data sets) and other races (Europe, 7 data sets; Oceania, 2 data sets) (ORs of rs599839: 1.31, 1.16, and 1.04, respectively).

\section{Table 2. Main results of rs599839 in the total and subgroup analysis.}

\begin{tabular}{|c|c|c|c|c|c|c|c|}
\hline \multirow[t]{2}{*}{ Race } & \multicolumn{2}{|l|}{ Total } & \multicolumn{2}{|c|}{ Heterogeneity } & \multirow[t]{2}{*}{ Model } & \multicolumn{2}{|c|}{ Bias in total } \\
\hline & OR $(95 \% \mathrm{CI})$ & $\mathrm{P}$ value & $Q_{\mathrm{x}}(\mathrm{P})$ & $I^{2}$ & & $P$ value for Begg test & $\mathrm{P}$ value for Egger test \\
\hline Total: A vs G & $1.17(1.07-1.28)$ & $0.0001^{*}$ & $32.42(0.001)$ & $69.2 \%$ & $\mathrm{RE}$ & 0.484 & 0.439 \\
\hline Europe: A vs G & $1.16(1.03-1.31)$ & $0.0001^{*}$ & $10.30(0.003)$ & $72.3 \%$ & RE & 0.573 & 0.550 \\
\hline Asia: A vs G & $1.31(1.17-1.47)$ & $0.0001^{*}$ & $3.74(0.154)$ & $46.6 \%$ & $\mathrm{FE}$ & 0.602 & 0.315 \\
\hline Oceania: $\mathrm{A} v s \mathrm{G}$ & $1.04(0.95-1.14)$ & 0.3900 & $0.77(0.380)$ & & $\mathrm{FE}$ & 0.317 & \\
\hline
\end{tabular}

$\mathrm{FE}=$ fixed-effect model $\mathrm{RE}=$ random-effect model. $*$ The pooled OR has a statistically significant.

\section{Sensitivity analysis}

We performed sensitivity analyses by excluding the studies that were not in HWE, and the pooled ORs were not materially altered using the recessive genetic model (data not shown), indicating that the model results were statistically robust. In the subgroup analyses, sensitivity analyses could not be performed because of the smaller sample size and lower power.

\section{Publication bias}

Begg's rank correlation and Egger's weighted regression methods were utilized to assess the publication bias in the recessive genetic model. The funnel plot is shown in Figure 4. No evidence for publication bias was found in our study.

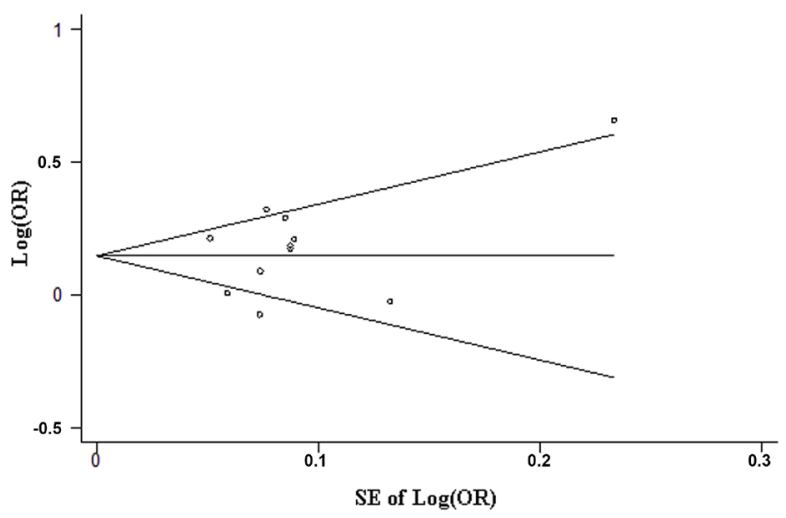

Figure 4. Funnel plot of rs599839. $\mathrm{SE}=$ standard error; $\mathrm{OR}=$ odds ratio.

\section{DISCUSSION}

The region with strongest association with CAD after 9p21 is the CELSR2-PSRC1SORT1 gene cluster. The risk allele at the CELSR2-PSRC1-SORT1 cluster has a frequency of $81 \%$ in European subjects and an additive OR of 1.19 (MIGen Consortium et al., 2009). rs646776 and 
rs599839 in the 1p13.3 region have been confirmed to exhibit correlation with CAD in a largescale analysis (Samani et al., 2007; Carrasquillo et al., 2010). Based on previous studies, evidence for the association between heart disease and chromosome 1p13.3 SNP markers exhibits strong credibility. The 1p13.3 SNP markers have been identified through GWAS and appear independent of traditional risk factors or family history (Samani et al., 2007). Our study investigated the two associated SNPs rs599839 (11 studies, $\mathrm{OR}=1.17$ ) and rs646776 (4 studies, $\mathrm{OR}=1.13$ ).

The genomic region at $1 \mathrm{p} 13.3$ contains four genes: proline/serine-rich coiled protein 1 (PSRC1), cadherin EGF LAG seven pass G-type receptor 2 (CELSR2), myosin-binding protein H-like (MYBHL), and sortilin 1 (SORT1). Of these, SORT1 has emerged as the most likely candidate causal gene. SORT1 is a transmembrane protein receptor that binds a variety of ligands and is involved in the endocytosis and degradation of lipoprotein lipase, a rate-limiting enzyme for the hydrolysis of triglycerides in lipoproteins (Nielsen et al., 1999). One recent expression analysis conducted using 400 liver samples expanded the association between the GWAS risk allele and increased LDL-C concentrations to decreases in both SORT1 and CELSR2 expression levels (Schadt et al., 2008). Another transcriptome analysis conducted using 176 whole blood samples suggested that functional variants at this locus might reside within the SORT1 gene since the risk allele was associated with decreased SORT1 expression, increased plasma LDL-C concentration, and increased CAD risk (Linsel-Nitschke et al., 2010). Because of the important role of LDL in the development of CVD, it had been assumed that increased SORT1 expression in carriers of the $\mathrm{G}$ allele led to greater LDL tissue uptake, which resulted in reduced circulating LDL levels and, subsequently, a lower CAD risk (Erdmann et al., 2010).

However, as most studies have indicated only a modest reduction in LDL-C in carriers of the homozygous GG genotype of SNP rs599839, it is possible that this allele does not entirely explain the significant reduction in the risk of CAD that has been observed for this SNP. In subsequent studies, we hope to further research the 1p13.3 region SORT1 gene and its relationship with LDL on a larger scale, to identify the exact evidence. Meanwhile, additional analyses will be required to exclude causal roles for other genes (i.e., CELSR2 and PSRC1) that map closer to the strongest associations across a neighboring recombination hotspot to SORT1. Recently, SORT1 has been connected to the endocytosis of ApoA-V-containing chylomicrons (Nilsson et al., 2008). The main SNP near SORT1 gene is associated with LDL-C and $\mathrm{ApoB}$, and this association is most significant in the whole genome scan. Similarly, the sampled variation at the locus as a whole is second only to variation near the APOE locus for explaining the variance in the lipids due to genetics (Chasman et al., 2008). In our separate data study, ApoA and ApoB were introduced into the final calibration model as covariates, and SNP rs599839 remained statistically significant. The final analysis showed that $1 \mathrm{p} 13$ and ApoA had a statistically significant interaction and worth further exploration.

Few studies have examined the associations between SNPs on chromosome 1p13.3 and CVD risk in Asian populations. Our research found that SNP rs599839 showed a higher association with the CVD $(\mathrm{OR}=1.31)$ in Asians. Such an association with lipid concentrations has been previously reported in a Japanese and Korean population (Xie et al., 2011). An even larger-scale case-control study in the Han Chinese population should be performed to evaluate the association of rs599839 and rs646776 with CAD susceptibility and serum lipid levels. Overall, our results suggest that the region of 1 p13.3 plays a role in multiple complex diseases. Further studies should focus on the identification of the underlying mechanism at this locus for CVD. In addition, the interactions between gender, smoking, and excess weight and these SNPs need to be replicated in other populations. 
This broad replication provided unprecedented evidence for association between genetic variants at chromosome 1p13.3 and the risk of CAD. The SNPs rs599839 and rs646776 might serve as novel genetic markers for CAD risk.

\section{Conflicts of interest}

The authors declare no conflict of interest.

\section{ACKNOWLEDGMENTS}

Research supported by Beijing Natural Science Foundation (\#7131002); Key Projects in the National Science \& Technology Pillar Program in the Twelfth Five-year Plan Period of China (\#2011BAI08B01); Beijing Municipal Natural Science Foundation (Serial \#7122016). The funders had no role in study design, data collection and analysis, decision to publish, or preparation of the manuscript.

\section{REFERENCES}

Aulchenko YS, Ripatti S, Lindqvist I, Boomsma D, et al. (2009). Loci influencing lipid levels and coronary heart disease risk in 16 European population cohorts. Nat. Genet. 41: 47-55.

Carrasquillo MM, Nicholson AM, Finch N, Gibbs JR, et al. (2010). Genome-wide screen identifies rs646776 near sortilin as a regulator of progranulin levels in human plasma. Am. J. Hum. Genet. 87: 890-897.

Chasman DI, Paré G, Zee RY, Parker AN, et al. (2008). Genetic loci associated with plasma concentration of LDL-C, HDLC, triglycerides, ApoA1, and ApoB among 6382 Caucasian women in genome-wide analysis with replication. Circ. Cardiovasc. Genet. 1: 21-30.

Ellis KL, Frampton CM, Pilbrow AP, Troughton RW, et al. (2011). Genomic risk variants at 1p13.3, 1q41, and 3q22.3 are associated with subsequent cardiovascular outcomes in healthy controls and in established coronary artery disease. Circ. Cardiovasc. Genet. 4: 636-646.

Erdmann J, Linsel-Nitschke P and Schunkert H (2010). Genetic causes of myocardial infarction: new insights from genome-wide association studies. Dtsch. Arztebl. Int. 107: 694-699.

Esparragón FR, Companioni O, Bello MG, Ríos NB, et al. (2012). Replication of relevant SNPs associated with cardiovascular disease susceptibility obtained from GWAs in a case-control study in a Canadian population. Dis. Markers 32: 231-239.

Gigante B, Leander K, Vikström M, Ye S, et al. (2012). Chromosome 1p13 genetic variants antagonize the risk of myocardial infarction associated with high ApoB serum levels. BMC Cardiovasc. Disord. 12: 90.

Guo J, Li W, Liu X, Wang XY, et al. (2011). Association of single-nucleotide polymorphisms on chromosome 1p13 and 9p21 with acute myocardial infarction in a Chinese population: the AMI study in China. Acad. J. Sec. Milit. Med. Univ. 31: 822-829.

Helgadottir A, Thorleifsson G, Manolescu A, Gretarsdottir S, et al. (2007). A common variant on chromosome 9p21 affects the risk of myocardial infarction. Science 316: 1491-1493.

Hobbs FD (2004). Cardiovascular disease: different strategies for primary and secondary prevention. Heart 90: 12171223.

Huang H, Pan L, Zhang L, Chen Y, et al. (2008). Association of single nucleotide polymorphism rs599839 on chromosome lpl3.3 with premature coronary heart disease in a Chinese Han population. Zhonghua Yi Xue Yi Chuan Xue Za Zhi 25: 686-689.

Karvanen J, Silander K, Kee F, Tiret L, et al. (2009). The impact of newly identified loci on coronary heart disease, stroke and total mortality in the MORGAM prospective cohorts. Genet. Epidemiol. 33: 237-246.

Kathiresan S, Melander O, Anevski D, Guiducci C, et al. (2008a). Polymorphisms associated with cholesterol and risk of cardiovascular events. N. Engl. J. Med. 358: 1240-1249.

Kathiresan S, Melander O, Guiducci C, Surti A, et al. (2008b). Six new loci associated with blood low-density lipoprotein cholesterol, high-density lipoprotein cholesterol or triglycerides in humans. Nat. Genet. 40: 189-197.

Kleber ME, Renner W, Grammer TB, Linsel-Nitschke P, et al. (2010). Association of the single nucleotide polymorphism 
rs599839 in the vicinity of the sortilin 1 gene with LDL and triglyceride metabolism, coronary heart disease and myocardial infarction the Ludwigshafen Risk and Cardiovascular Health Study. Atherosclerosis 209: 492-497.

Lee JY, Lee BS, Shin DJ, Woo Park K, et al. (2013). A genome-wide association study of a coronary artery disease risk variant. J. Hum. Genet. 58: 120-126.

Linsel-Nitschke P, Heeren J, Aherrahrou Z, Bruse P, et al. (2010). Genetic variation at chromosome 1p13.3 affects sortilin mRNA expression, cellular LDL-uptake and serum LDL levels which translates to the risk of coronary artery disease. Atherosclerosis 208: 183-189.

McPherson R, Pertsemlidis A, Kavaslar N, Stewart A, et al. (2007). A common allele on chromosome 9 associated with coronary heart disease. Science 316: 1488-1491.

Muendlein A, Geller-Rhomberg S, Saely CH, Winder T, et al. (2009). Significant impact of chromosomal locus 1p13.3 on serum LDL cholesterol and on angiographically characterized coronary atherosclerosis. Atherosclerosis 206: 494-499.

Myocardial Infarction Genetics Consortium, Kathiresan S, Voight BF, Purcell S, et al. (2009). Genome-wide association of early-onset myocardial infarction with single nucleotide polymorphisms and copy number variants. Nat. Genet. 41: 334-341.

Nielsen MS, Jacobsen C, Olivecrona G, Gliemann J, et al. (1999). Sortilin/neurotensin receptor-3 binds and mediates degradation of lipoprotein lipase. J. Biol. Chem. 274: 8832-8836.

Nilsson SK, Christensen S, Raarup MK, Ryan RO, et al. (2008). Endocytosis of apolipoprotein A-V by members of the low density lipoprotein receptor and the VPS10p domain receptor families. J. Biol. Chem. 283: 25920-25927.

Palomaki GE, Melillo S and Bradley LA (2010). Association between 9p21 genomic markers and heart disease: a metaanalysis. JAMA 303: 648-656.

Samani NJ, Erdmann J, Hall AS, Hengstenberg C, et al. (2007). Genomewide association analysis of coronary artery disease. N. Engl. J. Med. 357: 443-453.

Samani NJ, Braund PS, Erdmann J, Götz A, et al. (2008). The novel genetic variant predisposing to coronary artery disease in the region of the PSRC1 and CELSR2 genes on chromosome 1 associates with serum cholesterol. J. Mol. Med. 86: $1233-1241$.

Sandhu MS, Waterworth DM, Debenham SL, Wheeler E, et al. (2008). LDL-cholesterol concentrations: a genome-wide association study. Lancet 371: 483-491.

Schadt EE, Molony C, Chudin E, Hao K, et al. (2008). Mapping the genetic architecture of gene expression in human liver. PLoS Biol. 6: e107.

Teo KK, Liu L, Chow CK, Wang X, et al. (2009). Potentially modifiable risk factors associated with myocardial infarction in China: the INTERHEART China study. Heart 95: 1857-1864.

van Wyk JT, van Wijk MA, Sturkenboom MC, Moorman PW, et al. (2005). Identification of the four conventional cardiovascular disease risk factors by Dutch general practitioners. Chest 128: 2521-2527.

Wang AZ, Li L, Zhang B, Shen GQ, et al. (2011). Association of SNP rs17465637 on chromosome 1q41 and rs599839 on 1p13.3 with myocardial infarction in an American Caucasian population. Ann. Hum. Genet. 75: 475-482.

Wang TJ, Gona P, Larson MG, Tofler GH, et al. (2006). Multiple biomarkers for the prediction of first major cardiovascular events and death. N. Engl. J. Med. 355: 2631-2639.

Wellcome Trust Case Control Consortium (2007). Genome-wide association study of 14,000 cases of seven common diseases and 3,000 shared controls. Nature 447: 661-678.

Willer CJ, Sanna S, Jackson AU, Scuteri A, et al. (2008). Newly identified loci that influence lipid concentrations and risk of coronary artery disease. Nat. Genet. 40: 161-169.

Xie F, Chu X, Wu H, Sun W, et al. (2011). Replication of putative susceptibility loci from genome-wide association studies associated with coronary atherosclerosis in Chinese Han population. PLoS One 6: e20833. 